\title{
Two sliding mode control approaches for the stator voltage amplitude regulation of a stand-alone WRSM
}

\author{
A. Dòria-Cerezo, V.I. Utkin, R.S. Muñoz-Aguilar and E. Fossas
}

\begin{abstract}
In this paper two sliding mode control alternatives to regulate the stator voltage amplitude for a stand alone wound rotor synchronous generator are presented. Both controllers use the stator voltage d-component error in the sliding surface. In a first case an outer PI loop controller is added to provide the proper d-voltage component reference. The second approach consists in extending the dynamic system to include the integral term as state variable and to modify the former sliding surface by adding this new state. Finally, simulations results are done in order to validate the proposed algorithms.
\end{abstract}

\section{INTRODUCTION}

The wound rotor synchronous machine (WRSM) is a doubly-fed electrical machine which can be used for both: generation [1][2] and driving applications [3][4]. Usually, wound rotor synchronous machines, used in generation purposes, are studied when they are directly connected to the power grid [1]. In this case, the stator voltage and frequency are established by the power grid, while the rotor voltage helps to improve the power factor and to compensate the reactive power at the connection point. In this paper we study the isolated load case which is significantly different: neither amplitude nor frequency of stator voltage are fixed. For the stand-alone configuration, although the mechanical speed determines the frequency, the rotor voltage is used to set the stator amplitude.

The WRSM is controlled by several techniques, in industry the most common are based linear techniques [5][6], however, decoupling methods [7], widely employed for asynchronous machines, are also extended to the synchronous case. Modern non-linear techniques such as passivity-based control [3], are also used for regulating this kind of machine.

Several controllers for synchronous machines have also been obtained using sliding modes [8]. For example, the classical approach of the Sliding Mode Control (SMC) was used in [9] for a position servo system. More recently, sliding observers combined with control linearization and singular perturbations, are applied in [1] for a synchronous generator connected to a power grid. In [10] higher order sliding modes have been also proposed in order to avoid chattering problem for power control of a wind energy generator module.

\footnotetext{
A. Dòria-Cerezo and R.S. Muñoz-Aguilar are with the Department of Electrical Engineering and the Institute of Industrial and Control Engineering, Universitat Politècnica de Catalunya, Spain arnau.doriadupc.edu, raul.munoz-aguilareupc.edu

V.I. Utkin is with the Department of Electrical Engineering, The Ohio State University, OH 43210, USA utkin@ece. osu. edu

E. Fossas is with the Department of Automatic Control and the Institute of Industrial and Control Engineering, Universitat Politècnica de Catalunya, Spain enric.fossastupc.edu
}

The main goal of this work is to design a sliding mode control algorithm for a wound rotor synchronous generator feeding an isolated resistive unknown load.

In this case, SMC is not directly applied to the system natural output (stator voltage amplitude). Instead, the sliding surface takes into account the $\mathrm{d}$ component of the stator voltage. Actually, the sliding surface is the error between that $d$ component and a reference, presumed that all the coefficients are known. An integral term makes the system robust in front of the parameter variation.

The paper is organized as follows. First, the system and its dynamics are introduced in Section II. In Section III the sliding mode controller with an external PI loop is designed. The use of a dynamic extension allows to design a different control law, where the integral term is directly considered in the sliding surface. This is presented in Section IV. Simulation results are shown in Section V and, finally, conclusions are stated in Section VI.

\section{SYSTEM DESCRIPTION}

Figure 1 shows the proposed scenario: a primary mover (for example an Internal Combustion Engine, ICE) drags a WRSM, which acts as a generator to fed an isolated load. $\omega_{m}$ is the mechanical speed, $v_{s}, i_{s}$ are the vectors corresponding to the stator voltages and currents, and $v_{F}, i_{F}$ are the field voltage and current.

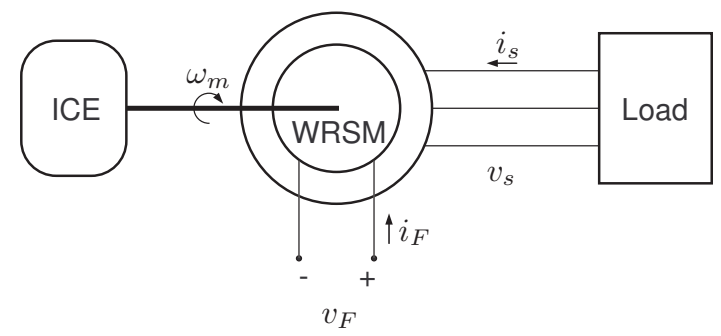

Fig. 1. Scheme of a stand-alone wound rotor synchronous generator.

As explained before, this system is different from the typical grid connection where the frequency and the voltage amplitudes are fixed by the grid. For an isolated connection, the frequency is determined by the mechanical speed (provided by the ICE), while the voltage amplitude must be assured by the rotor field voltage. 


\section{A. Dynamic model}

From the well-known dynamical equations (in the $\mathrm{dq}$ coordinates) of the WRSM, and the interconnection rules with a pure resistor load, the whole dynamical system is presented.

Assuming that the mechanical speed is externally regulated by the ICE, the electrical part of the wound rotor synchronous machine can be described as

$$
L \frac{\mathrm{d} x}{\mathrm{~d} t}=\left(\begin{array}{ccc}
-R_{s} & \omega L_{s} & 0 \\
-\omega L_{s} & -R_{s} & -\omega L_{m} \\
0 & 0 & -R_{F}
\end{array}\right) x+\left(\begin{array}{c}
v_{d} \\
v_{q} \\
v_{F}
\end{array}\right)
$$

where

$$
L=\left(\begin{array}{ccc}
L_{s} & 0 & L_{m} \\
0 & L_{s} & 0 \\
L_{m} & 0 & L_{F}
\end{array}\right)
$$

is the inductance matrix, $x^{T}=\left(i_{d}, i_{q}, i_{F}\right) \in \mathbb{R}^{3}$ are the dqstator and field currents, $R_{s}$ and $R_{F}$ are the stator and field resistances, $L_{s}, L_{m}$ and $L_{F}$ are the stator, mutual and field inductances, $\omega$ is the electrical speed $\left(\omega=n_{p} \omega_{m}\right.$, where $n_{p}$ is the number of pole pairs), $v_{d}, v_{q}$ and $v_{F}$ are the dq-stator voltages and the field voltage which will be used as a control input.

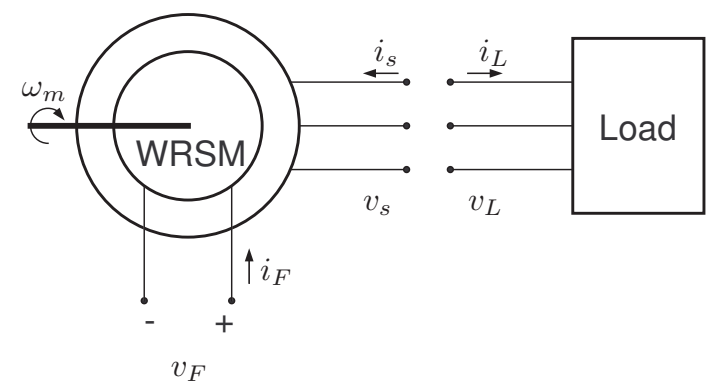

Fig. 2. Detail of the interconnection between a wound rotor synchronous machine and a load.

In order to design the control law, let us obtain the complete model of a WRSM connected to a resistive load $R_{L}$. The interconnection scheme is depicted in Figure 2. $v_{L}^{T}=\left(v_{L d}, v_{L q}\right) \in \mathbb{R}^{2}$ and $i_{L}^{T}=\left(i_{L d}, i_{L q}\right) \in \mathbb{R}^{2}$ are the dq load voltages and currents, which are related by

$$
\left(\begin{array}{c}
v_{L d} \\
v_{L q}
\end{array}\right)=R_{L}\left(\begin{array}{c}
i_{L d} \\
i_{L q}
\end{array}\right)
$$

where $R_{L}$ is the resistance value. According to Figure 2, the interconnection rules are

$$
\begin{aligned}
& v_{s}=v_{L} \\
& i_{L}=-i_{s} .
\end{aligned}
$$

Now, putting together (1) and (2), the system can be written in an affine form as

$$
L \frac{\mathrm{d} x}{\mathrm{~d} t}=A x+B v_{F},
$$

where $L$ is the inductance matrix defined before,

$$
A=\left(\begin{array}{ccc}
-\left(R_{s}+R_{L}\right) & \omega L_{s} & 0 \\
-\omega L_{s} & -\left(R_{s}+R_{L}\right) & -\omega L_{m} \\
0 & 0 & -R_{F}
\end{array}\right),
$$

and

$$
B=\left(\begin{array}{l}
0 \\
0 \\
1
\end{array}\right)
$$

\section{B. Equilibrium points}

The equilibrium points can be parametrized by the control input $v_{F}$, this resulting in

$$
x^{*}=-A^{-1} B v_{F},
$$

which are on a straight line defined by

$$
x^{* T}\left(v_{F}\right)=\left[-\frac{\omega^{2} L_{s} L_{m}}{R_{F}\left|Z_{s}\right|^{2}},-\frac{\omega L_{m}\left(R_{s}+R_{L}\right)}{R_{F}\left|Z_{s}\right|^{2}}, \frac{1}{R_{F}}\right] v_{F}
$$

where $\left|Z_{s}\right|^{2}=\omega^{2} L_{s}^{2}+\left(R_{s}+R_{L}\right)^{2}$.

\section{Control objective}

As mentioned before, this machine must ensure stator voltage amplitude and frequency. For a synchronous machine, the stator frequency is directly given by the mechanical speed, which, in this paper is assumed to be constant and externally regulated. Then, the system output is the stator voltage amplitude $V_{s}$, which can be easily obtained, in a dq-framework as

$$
V_{s}=\sqrt{v_{d}^{2}+v_{q}^{2}}
$$

Last equation can be expressed in current terms using (2), which yields

$$
V_{s}=R_{L} \sqrt{i_{d}^{2}+i_{q}^{2}}
$$

and the control input is the field voltage $v_{F}$.

Note that, a fix value of $V_{s}$ in (6), namely $V_{s}=V_{\text {ref }}$,

$$
V_{\text {ref }}^{2}=R_{L}^{2}\left(i_{d}^{2}+i_{q}^{2}\right),
$$

implies that the control goal defines a cylinder in the state space, see Figure 3.

Then, the desired equilibrium points are the intersection of the straight line (4) of the WRSM and the cylinder (7). Using polar coordinates

$$
\begin{aligned}
& i_{d}=I_{s} \cos \delta \\
& i_{q}=I_{s} \sin \delta
\end{aligned}
$$

where, $I_{s}=\frac{V_{s}}{R_{L}}$, it is easy to obtain

$$
\begin{aligned}
i_{d}^{*} & =\frac{V_{\text {ref }}}{R_{L}} \cos \delta^{*} \\
i_{q}^{*} & =\frac{V_{\text {ref }}}{R_{L}} \sin \delta^{*} \\
i_{F}^{*} & =-\frac{V_{r e f}}{R_{L}} \frac{L_{s}}{L_{m} \cos \delta^{*}}
\end{aligned}
$$

where

$$
\delta^{*}=\arctan \left(\frac{R_{s}+R_{L}}{\omega L_{s}}\right) .
$$


In fact, there exist two solutions, given by the two values of the arctan function, which are depicted in Figure 3.

Furthermore, the value of the field voltage in equilibria is

$$
v_{F}^{*}= \pm \frac{R_{F} L_{s}}{R_{L} L_{m} \cos \delta^{*}} V_{r e f} .
$$

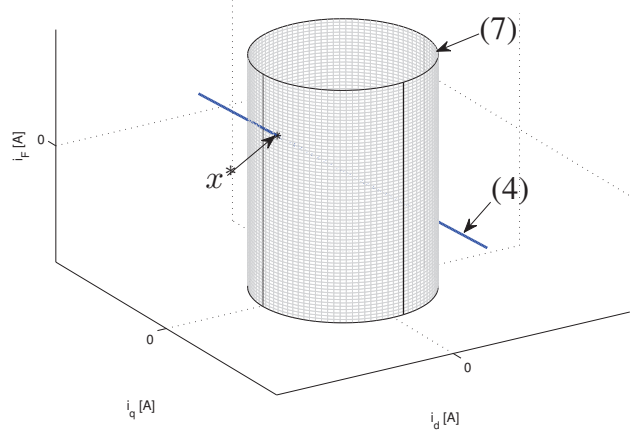

Fig. 3. Intersection of the control goals and the equilibrium points distribution of the system.

\section{SLIDING Mode CONTROL With AN OUTER-PI LOOP}

Figure 4 shows the proposed control algorithm based on two loops; an inner-loop based on SMC and a PI outer loop which provides the d-component of the stator voltage reference, $v_{d}^{r e f}$, to reach $V_{\text {ref }}$. Roughly speaking, when the switching system is faced with a perturbation, the PI loop places the sliding surface in the appropriate regulation point. This case differs from the one will be presented in Section IV, where the integral term is considered as a new state.

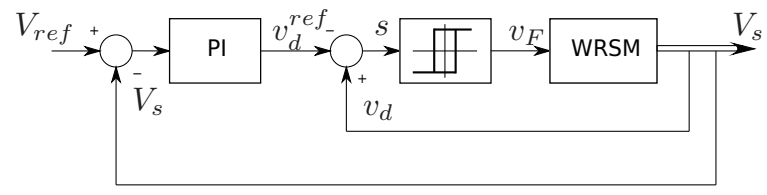

Fig. 4. Control scheme for a wound rotor synchronous generator.

The whole stability proof is based on the assumption of a fast inner-loop, with respect to the PI dynamics.

\section{A. Inner-loop: a sliding mode controller}

The Sliding Mode Controller enforces the system trajectories to reach and keep on the sliding surface, $s(x)=0$, with the switching function

$$
s(x)=v_{d}(x)-v_{d}^{r e f},
$$

where $v_{d}^{r e f}$ is defined by the outer-loop PI controller. From (2),

$$
s(x)=R_{L} i_{d}-v_{d}^{r e f} .
$$

Note that $s(x)=0$ defines the plane in $i_{d}=\frac{v_{d}^{r e f}}{R_{L}}$. This case differs from [2], where the sliding surface was the cylinder (7). The equivalent control, $u_{e q}$ is the solution of

$$
\frac{\partial s}{\partial x} L^{-1}\left(A x+B u_{e q}\right)=0 .
$$

It results in

$$
u_{e q}=\frac{1}{L_{m}}\left(-L_{F}\left(R_{s}+R_{L}\right) i_{d}+\omega L_{s} L_{F} i_{q}+L_{m} R_{F} i_{F}\right) .
$$

There is sliding motion on $s(x)=0$ provided that the reachability condition $s \cdot \frac{\mathrm{d} s}{\mathrm{~d} t}<0$ holds. From (3)

$$
s \cdot \frac{\mathrm{d} s}{\mathrm{~d} t}=s \frac{\partial s}{\partial x} L^{-1}\left(A x+B v_{F}\right)<0
$$

that, using (3) and (11), can be written as

$$
-s \frac{R_{L} L_{m}}{\mu}\left(v_{F}-u_{e q}\right)<0,
$$

finally, as $R_{L}, L_{m}, \mu>0$, the reachability condition yields

$$
s\left(u_{e q}-v_{F}\right)<0 .
$$

Using a bang-bang rotor voltage $\left(v_{F}= \pm V_{D C}\right)$, the following switching policy

$$
v_{F}=\left\{\begin{array}{lll}
V_{D C} & \text { if } & s>0 \\
-V_{D C} & \text { if } & s<0
\end{array}\right.
$$

fulfils the reachability condition presumed that $-V_{D C}<$ $u_{e q}<V_{D C}$.

The remaining dynamics (ISD) results in the following linear system

$$
\begin{aligned}
\frac{\mathrm{d} i_{q}}{\mathrm{~d} t} & =-\frac{R_{s}+R_{L}}{L_{s}} i_{q}-\frac{\omega L_{m}}{L_{s}} i_{F}-\frac{\omega}{R_{L}} v_{d}^{r e f} \\
\frac{\mathrm{d} i_{F}}{\mathrm{~d} t} & =\frac{\omega L_{s}}{L_{m}} i_{q}-\frac{R_{s}+R_{L}}{L_{m} R_{L}} v_{d}^{r e f},
\end{aligned}
$$

which is stable because the plant parameters are positive.

\section{B. Outer-loop: a PI controller}

The outer-loop consists in a simple PI controller. Considering a fast inner-loop, the closed-loop system, (3) and (12), reduces to (13) and (14), where the new input is $v_{d}^{\text {ref }}$ and the output still is $V_{s}$. Though the output remains nonlinear, presuming the ideal sliding dynamics achieves the steadystate, (5) can be rewritten as

$$
V_{s}=\sqrt{\left(v_{d}^{r e f}\right)^{2}+R_{L}^{2} i_{q}^{2}}
$$

Linearizing around $\left(v_{d}^{r e f}, i_{q}^{*}\right)$ such that

$$
V_{\text {ref }}=\sqrt{\left(v_{d}^{r e f *}\right)^{2}+R_{L}^{2} i_{q}^{* 2}},
$$

equation (15) results in

$$
V_{s} \simeq V_{r e f}+\frac{v_{d}^{r e f *}}{V_{r e f}}\left(v_{d}^{r e f}-v_{d}^{r e f *}\right)+\frac{R_{L}^{2} i_{q}^{*}}{V_{r e f}}\left(i_{q}-i_{q}^{*}\right)
$$

where

$$
v_{d}^{r e f *}=V_{\text {ref }} \cos \delta^{*} .
$$


Using (9), (16) and (18), equation (17) can be rewritten as

$$
V_{s} \simeq v_{d}^{r e f} \cos \delta^{*}+R_{L} i_{q} \sin \delta^{*} .
$$

Note that (19), which results from linearising (15) is a function of $\left(v_{d}^{r e f}, i_{q}\right)$ but it is independent of the voltage component of the point around which function $V_{s}$ was linearised.

Using (13) and (14) in (19) the transfer function $\frac{V_{s}}{v_{d}^{\text {ref }}}$ is obtained. Namely ${ }^{1}$,

$$
G(s)=\frac{\cos ^{2} \delta^{*} s^{2}+\omega^{2}}{\cos \delta^{*} s^{2}+\omega \sin \delta^{*} s+\omega^{2} \cos \delta^{*}} .
$$

The closed loop system transfer function is given by

$$
W(s)=\frac{c_{3} s^{3}+c_{2} s^{2}+c_{1} s+c_{0}}{b_{3} s^{3}+b_{2} s^{2}+b_{1} s+b_{0}}
$$

where $c_{3}=k_{p} \cos ^{2} \delta^{*}, c_{2}=k_{i} \cos ^{2} \delta^{*}, c_{1}=k_{p} \omega^{2}, c_{0}=$ $k_{i} \omega^{2}$ and

$$
\begin{aligned}
& b_{3}=\cos \delta^{*}\left(k_{p} \cos \delta^{*}+1\right) \\
& b_{2}=\left(\omega \sin \delta^{*}+k_{i} \cos ^{2} \delta^{*}\right) \\
& b_{1}=\omega^{2}\left(k_{p}+\cos \delta^{*}\right) \\
& b_{0}=k_{i} \omega^{2} .
\end{aligned}
$$

Application of the Routh-Hurwitz criterion yields to the next stability conditions

$$
\begin{aligned}
k_{p} & >-\frac{1}{\cos \delta^{*}} \\
k_{i} & >0 \\
k_{i} & <\frac{\omega\left(k_{p} \sin \delta^{*}+\cos \delta^{*}\right)}{\cos \delta^{*} \sin \delta^{*}} .
\end{aligned}
$$

\section{Direct Sliding Mode Controller}

In this section, a direct Sliding Mode Controller is presented. The sliding surface is still based on the error of the d-component of the stator voltage, and an integral action is added in order to robustify the controller. Figure 5 shows the proposed control scheme.

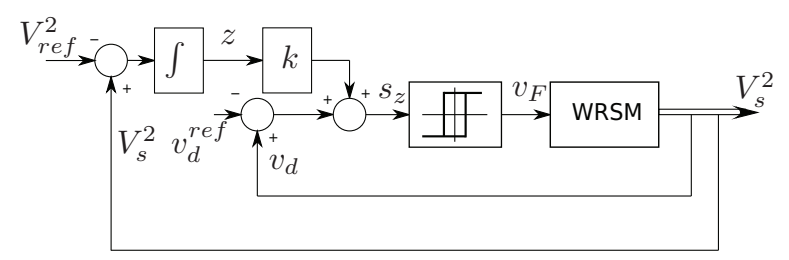

Fig. 5. Direct sliding mode control scheme for a wound rotor synchronous generator.

\footnotetext{
${ }^{1}$ This transfer function takes the form $G(s)=1+Q(s)$. Hence, a jump in $v_{d}^{r e f}$ results in a jump in a $V_{s}$. But this only occurs presuming ideal sliding dynamics. In a real application, $V_{D C}$ limitations and the own dynamics of the inner loop will filter the output signal $V_{s}$.
}

\section{A. Control design}

The integral term entails the extension of the system with a new variable $z$ defined by

$$
\frac{\mathrm{d} z}{\mathrm{~d} t}=V_{s}^{2}-V_{\text {ref }}^{2} .
$$

The new sliding surface, $s_{z}(x)=0$, is given by

$$
s_{z}=v_{d}-v_{d}^{r e f}+k z
$$

where $v_{d}^{\text {ref }}$ is the $v_{d}$ nominal value given $V_{\text {ref }} . v_{d}^{\text {ref }}$ is obtained from equations (8) and (2) using the nominal plant parameters $^{2}$

$$
v_{d}^{r e f}=V_{\text {ref }} \cos \tilde{\delta}^{*} .
$$

The equivalent control is obtained. It results in

$$
u_{z e q}=u_{e q}+\frac{\mu}{L_{m} R_{L}} k \dot{z}
$$

note that it equals to the former equivalent control plus the derivative of the new integral term.

Now, the control law is designed in the extended system

$$
L_{z} \frac{\mathrm{d} z_{e}}{\mathrm{~d} t}=A_{z}\left(z_{e}\right)+B_{z} v_{F}
$$

where $z_{e}^{T}=\left[i_{d}, i_{q}, i_{F}, z\right]$,

$$
\begin{gathered}
L_{z}=\left(\begin{array}{cccc}
L_{s} & 0 & L_{m} & 0 \\
0 & L_{s} & 0 & 0 \\
L_{m} & 0 & L_{F} & 0 \\
0 & 0 & 0 & 1
\end{array}\right), \\
A_{z}\left(z_{e}\right)=\left(\begin{array}{c}
A x \\
R_{L}^{2}\left(i_{d}^{2}+i_{q}^{2}\right)-V_{r e f}^{2}
\end{array}\right)
\end{gathered}
$$

and $B_{z}^{T}=(0,0,1,0)$. Tacking into account that $R_{L}, L_{m}, \mu>0$ and proceeding as usual, reachability condition yields

$$
s_{z}\left(u_{z e q}-v_{F}\right)<0,
$$

and, consequently, the switching control policy

$$
v_{F}=\left\{\begin{array}{lll}
V_{D C} & \text { if } \quad s_{z}>0 \\
-V_{D C} & \text { if } \quad s_{z}<0
\end{array}\right.
$$

guaranties sliding motion on $s_{z}=0$ presumed that $-V_{D C}<$ $u_{z e q}<V_{D C}$.

\section{B. Ideal Sliding Dynamics}

The controller defined in (21) ensures sliding modes on the switching surface. Using $s_{z}=0$, replacing $v_{F}=u_{z e q}$ in (20) and defining a new variable $\xi=v_{d}^{r e f}-k z$, the ideal sliding dynamics can be written as

$$
\begin{aligned}
\frac{\mathrm{d} i_{q}}{\mathrm{~d} t}= & -\frac{R_{s}+R_{L}}{L_{s}} i_{q}-\frac{\omega L_{m}}{L_{s}} i_{F}-\frac{\omega}{R_{L}} \xi \\
\frac{\mathrm{d} i_{F}}{\mathrm{~d} t}= & \frac{k L_{s} R_{L}}{\mu L_{m}} i_{q}^{2}+\frac{\omega L_{s}}{L_{m}} i_{q}+\frac{k L_{s}}{\mu L_{m} R_{L}} \xi^{2}-\frac{R_{s}+R_{L}}{L_{m} R_{L}} \xi \\
& -\frac{k L_{s}}{\mu L_{m} R_{L} R_{L}} V_{r e f}^{2} \\
\frac{\mathrm{d} \xi}{\mathrm{d} t}= & -\frac{k R_{L}^{2}}{\mu} i_{q}^{2}-\frac{k}{\mu} \xi^{2}+\frac{k}{\mu} V_{r e f}^{2} .
\end{aligned}
$$

${ }^{2}$ The tilde in $\tilde{\delta}^{*}$ denotes that this value depends on $R_{L}, R_{s}$ and $L_{s}$ estimations. 
As for the local stability, the small signal model around the equilibrium point given by (9), (10) and

$$
\xi^{*}=V_{\text {ref }} \cos \delta^{*}
$$

is used. This yields a sufficient condition for $k$. Namely ${ }^{3}$, $k>0$.

\section{Simulations}

In this Section the simulation results using the designed controller are presented. The used WRSM is a 4 poles, $2.4 \mathrm{~kW}$ machine with the following parameters: $R_{s}=3.06 \Omega$, $L_{s}=0.48 \mathrm{H}, L_{m}=0.31 \mathrm{H}, R_{F}=2.48 \Omega, L_{F}=0.24 \mathrm{H}$. Mechanical speed is set to $\omega_{m}=1500 \mathrm{rpm}$ and $V_{D C}=35 \mathrm{~V}$.

Initial conditions are $V_{\text {ref }}=200 \sqrt{2} \mathrm{~V}$ with a resistive load $R_{L}=120 \Omega$. Simulation results show the closed loop response when the load varies from the nominal value to $R_{L}=64 \Omega$ at $t=0.05 \mathrm{~s}$. The controller gains are: $k_{p}=$ $1000, k_{i}=100$ (for the PI controller) and $k=0.2$ (for the direct scheme). Simulations have been performed using a variable step integration and a zero order holder of sampling frequency $\omega_{s}=10 \mathrm{kHz}$.

Figure 6 shows the three phase stator voltages. In both cases, regulation of the stator voltage amplitude is achieved. The fast response of the first controller algorithm is a consequence of the proportional action of the PI loop.
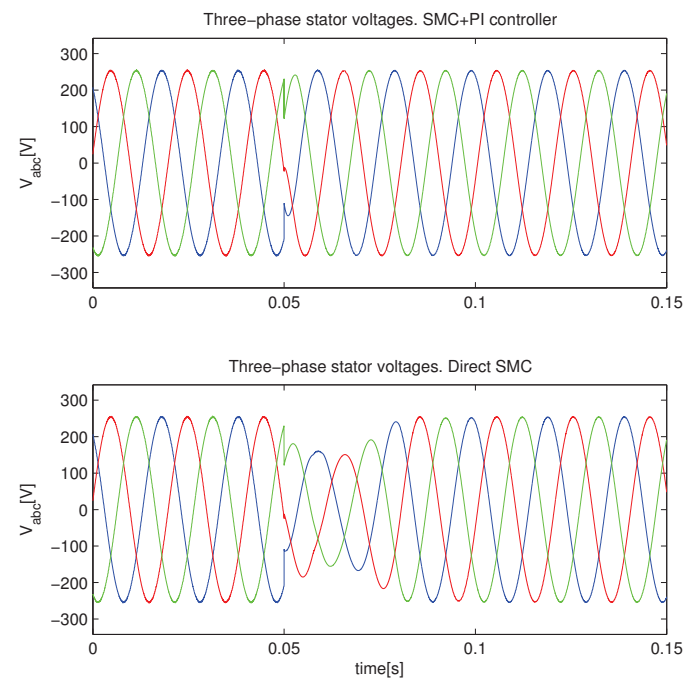

Fig. 6. Simulation results: Three phase stator voltages.

Figure 7 shows the switching functions. Note that in the second case, sliding is lost twice for a short time after the load change. The first time is due to the sudden load variation, while the second time is caused by the equivalent control (11) which is out of range $\left(\left|u_{z e q}\right|>V_{D C}\right)$. See also

\footnotetext{
${ }^{3}$ As pointed out at the end of Section II, two equilibria are possible. To reach the value corresponding to $\delta^{*}=\arctan \left(\frac{R_{S}+R_{L}}{\omega L_{S}}\right)+\pi$, local stability condition also holds with $k<0$.
}

Figure 8 , where the field voltage $v_{F}$ is shown. Remark the long time the control variable remains saturated compared with the SMC+PI control law.
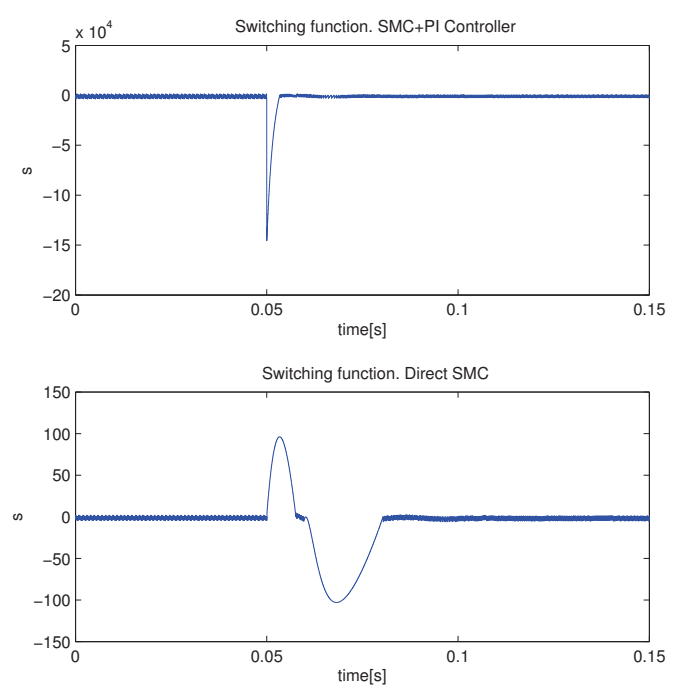

Fig. 7. Simulation results: Switching functions.
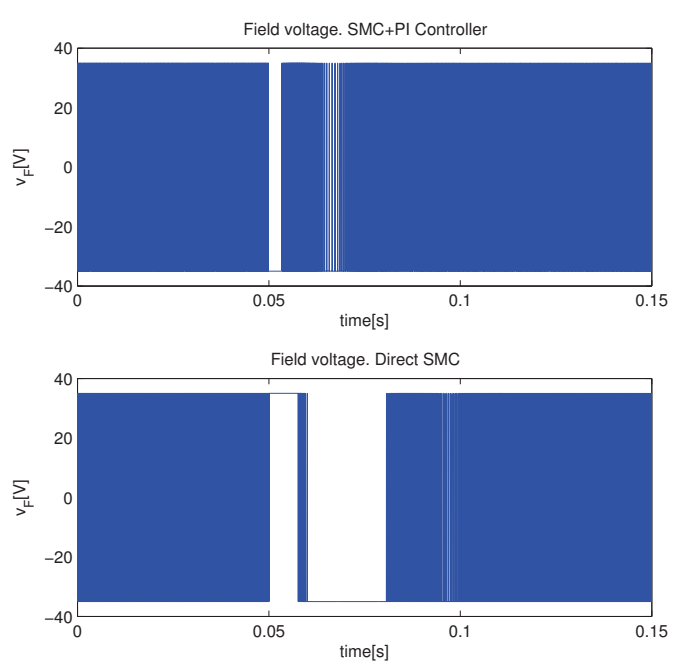

Fig. 8. Simulation results: Field voltages.

Velocity response can be observed in Figures 9 and 10, where stator voltage amplitude and stator and field currents are displayed. The faster response of the SMC+PI controller, in front of the direct scheme, is clear.

\section{CONCLUSIONS}

In this paper two approaches for controlling the stator voltage amplitude of an isolated wound rotor synchronous 
2010 11th International Workshop on Variable Structure Systems

Mexico City, Mexico, June 26 - 28, 2010

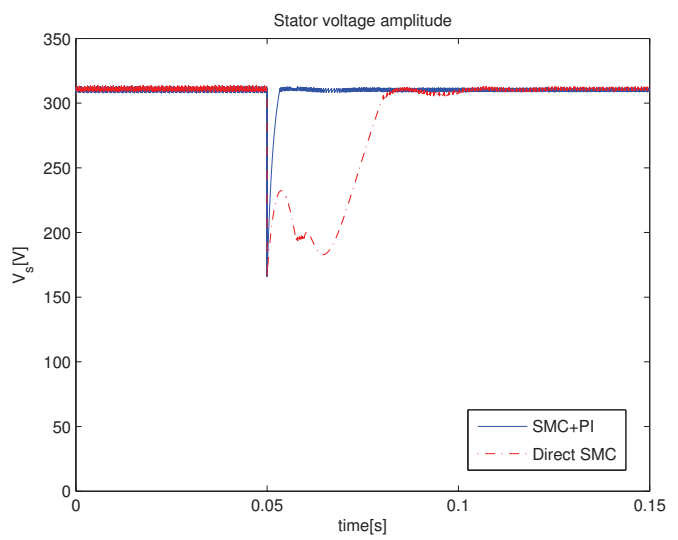

Fig. 9. Simulation results: Stator voltage amplitudes.
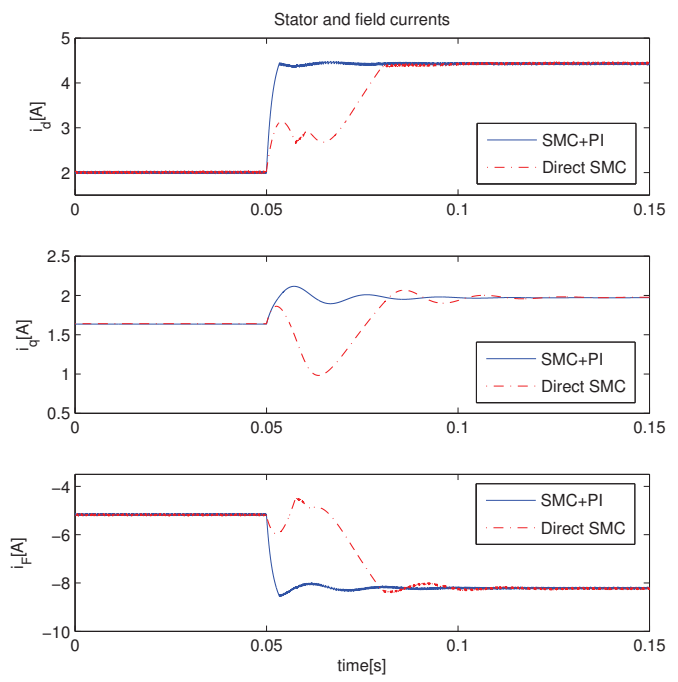

Fig. 10. Simulation results: Stator and field currents.

generator feeding a resistive load are presented. Both controllers are designed using sliding modes, and taking the error of the d-voltage component as switching function. First controller is composed by an inner sliding mode based loop, and a simple outer-loop PI controller, while the second approach suggest the use of a dynamic extension in the switching function.

Stability of both alternatives are studied. The inner sliding mode control loop is globally stable, while the design of the outer PI loop is based on the linearization of the ideal sliding dynamics. The stability of the second control algorithm is locally proved.

The control algorithms do not require the WRSM parameter knowledge; they only need the stator voltage measures and the rotor position. This allows to avoid current sensors and consequently, it reduces the implementation costs.

Simulation results shows that the two possibilities achieve the control goal. However, since the direct scheme does not contain any proportional gain in $V_{s}-V_{\text {ref }}$, step changes in $V_{\text {ref }}$ only appear in the switching function trough the integral term. On the contrary, the proportional part in the SMC+PI allows reflecting reference step changes in $s$ directly. The inclusion of the proportional part in the direct SMC implies the transversality condition fails at $i_{d}=0$, see [2].

Along this paper we assumed that the mechanical speed is externally regulated. In some cases where the ICE power is close to the load power, some variations of the mechanical speed can occur during the load transients, however this would not affect to the amplitude stator regulation since this speed remains positive. Stability is still guaranteed in both cases, and only the gains of the PI loop of the SMC+PI algorithm should be redesigned.

\section{ACKNOWLEDGMENTS}

A. Dòria-Cerezo and R. S. Muñoz-Aguilar were partially supported by the Spanish government research projects DPI2007-62582 and ENE2009-13998-C02-01, respectively. E. Fossas was partially supported by the Spanish government research projects DPI2007-62582 and DPI2008-01408.

\section{REFERENCES}

[1] J. Cabrera-Vázquez, A.G. Loukianov, J.M. Canedo, and V.I. Utkin. Robust controller for synchronous generator with local load via vsc. Electrical Power and Energy Systems, 29:348-359, 2007.

[2] A. Dòria-Cerezo, E. Fossas, R.S. Munoz-Aguilar, and V.I. Utkin. Sliding mode control of an isolated wound rotor synchronous generator. In Proc. of the 10th European Control Conference, 2009.

[3] C. Batlle, A. Dòria-Cerezo, and G. Espinosa. Simultaneous idapassivity-based control of a wound rotor synchronous motor. In Proc. IEEE Conf. on Decision and Control, pages 3187-3191, 2008.

[4] C. Rossi, D. Casadei, A. Pilati, and M. Marano. Wound rotor salient pole synchronous machine drive for electric traction. In Proc. IEEE Industry Applications Conference, 2006.

[5] W. Leonhard. Control of electric drives. Springer, 1995.

[6] M.K. Senesky, P. Tsao, and S.R. Sanders. Simplified modelling and control of a synchronous machine with variable-speed six-step drive. In Proc. IEEE Applied Power Electronics Conference and Exposition, 2004.

[7] E. Ho and P.C. Sen. High-performance decoupling control techniques for various rotating field machines. IEEE Trans. on Industrial Electronics, 42(1):40-49, 1995.

[8] V. Utkin, J. Guldner, and J. Shi. Sliding Mode Control in Electromechanical Systems. Taylor and Francis, 1999.

[9] C. Namuduri and P.C. Sen. A servo-control system using a selfcontrolled synchronous motor ( $\mathrm{scsm}$ ) with sliding mode controller. IEEE Trans. on Industry Applications, 23(2):283-295, 1987.

[10] F. Valenciaga and P.F. Puleston. High-order sliding control for a wind energy conversion system based on a permanent magnet synchronous generator. IEEE Trans. on Energy Conversion, 23(3), 2008. 